Independent pairs reviewed all cites and articles and extracted data, and narrative synthesis was carried out.

Results Our searches identified 1950 possible relevant articles, of which 27 were included for data extraction. Of these, 15 papers included definitions of case management, and 14 common elements were identified. Twenty two explained the role of case manager and in 18 a description of tasks was found. In 25 articles referral services were mentioned. The most common defining elements were 'return to work intervention', 'multidisciplinary assessment' and 'interdisciplinary intervention' $(53.8 \%)$. The $40 \%$ of the articles emphasized the 'coordination', 'to influence multiple factors' (33.3\%) and 'individual approach' (26.7\%). The tasks included 'to establish goals and plan the rehabilitation for return to work' $(50 \%)$, and 'supervise or coordinate return to work process and to offer/refer the employee to services/adaptations or therapeutic workplaces' (36.4\%) The most common offered services were mental health $(64.0 \%)$, and rehabilitation (48.0\%).

Conclusions Despite the increase in the number of published articles dealing with case management, usually it is not clearly defined. This scoping review emphasizes the need to define case management and its organizational characteristics, and proposes an extended and updated definition.

\section{A.2 MUSCULOSKELETAL SYMPTOMS AND WORK-RELATED ACCIDENTS: A HOSPITAL-BASED CASE-CONTROL STUDY}

${ }^{1}$ Adriano Dias*, ${ }^{1}$ João Marcos Bernardes, ${ }^{2,3}$ Juan Gomez-Salgado, ${ }^{2,3}$ Carlos Ruiz-Frutos. ${ }^{1}$ Unesp - São Paulo State University, Botucatu Medical School, Botucatu, Brazil; ${ }^{2}$ Universidad de Huelva, Huelva, Spain; ${ }^{3}$ Universidad Espíritu Santo, Guayaquil, Ecuador

\subsection{6/OEM-2019-EPI.111}

This hospital-based case-control study aimed to determine whether self-reports of musculoskeletal symptoms (MSS) were associated with the occurrence of work-related accidents. Study participants were recruited from the emergency department at Botucatu Medical School University Hospital. Cases were workers who suffered work-related accidents that required hospitalization, while controls were selected patients who suffered a non-work-related accident. Participants were interviewed using a standardized structured questionnaire with close-ended questions and a modified version of the Brazilian Portuguese Nordic Musculoskeletal Questionnaire. Associations between self-reports of MSS and work-related accidents were analyzed with two logistic regression models (one for symptoms that occurred in the 12 months period and the other for those that occurred in the previous 7 days). These analyses were performed in two steps: univariate and multiple model. Variables with a Pvalue $\leq 0.25$ in the univariate analysis were included in the multiple models, using the forward stepwise selection procedure. In the multiple models two-sided P-values less than 0.05 were considered statistically significant. Altogether, 80 cases and 125 controls were included. The participants had a mean age of 36.9 years $(\mathrm{SD}=11.4)$ and $72.2 \%$ were men. In the 12 months multiple logistic regression model, self-report of MSS in the upper limbs (OR 2.689 95\% CI 1.357-5.326) was associated with increased odds of workrelated accidents occurrence, while in the 7 days multiple logistic regression model, self-report of MSS in the upper limbs (OR 2.374 95\% CI 1.083-5.201) and in the vertebral column (OR 2.154 95\% CI 1.017-4.561) were associated. Thus, this case-control study suggests that MSS in the upper limbs and in the vertebral column are associated with increased odds of work-related accidents; and that the Nordic Musculoskeletal Questionnaire may be used as a complementary screening tool for identifying workers at risk for work-related accidents.

\section{A.3 INJURED AT WORK: FACTORS PREDICTIVE OF FURTHER WORK-RELATED INJURIES - A PROSPECTIVE STUDY}

${ }^{1}$ Helen Harcombe*, ${ }^{2}$ Ari Samaranayaka, ${ }^{3}$ Emma H Wyeth, ${ }^{1}$ Gabrielle Davie, ${ }^{4}$ lan D Cameron, ${ }^{1}$ Sarah Derrett. ${ }^{1}$ Injury Prevention Research Unit, Department of Preventive and Social Medicine, University Of Otago, New Zealand; ${ }^{2}$ Ng̈̈ Tahu Mæri Health Research Unit, Department of Preventive and Social Medicine, University of Otago, New Zealand; ${ }^{3}$ John Walsh Centre for Rehabilitation Research, Kolling Institute, University of Sydney, Australia; ${ }^{4}$ Biostatistics Unit, Dean's Department, University of Otago, New Zealand

\subsection{6/OEM-2019-EPI.112}

Background Work-related injuries can have a large impact on employees, employers and wider society. Preventing subsequent work-related injuries from occurring among those who have already had a work-related injury has the potential to reduce the burden of work-related injuries. However, predictors of subsequent work injuries must first be understood.

This study investigates New Zealand participants from the Prospective Outcomes of Injury Study (POIS) with a substantial work-related injury (the 'sentinel' injury) and examines subsequent work-related injury events occurring during the following 24 months. Objectives are to: 1) describe the nature of sentinel and subsequent work-related injuries, and 2) examine work-related predictors of subsequent workrelated injuries.

Methods Of the 2856 POIS participants, 754 were recruited following a substantial work-related injury. Data were combined from: 1) participant interviews approximately 3 months following their sentinel injury, 2) administrative claims data from New Zealand's universal no-fault injury insurer (the Accident Compensation Corporation), and 3) hospital discharge data to 24 months. Predictors of subsequent work-related injuries were examined using multivariable analyses.

Results Work-related sentinel injury events most commonly involved spine dislocations/sprains/strains (25\%) with 'lifting/ loading/pulling' the most common work task involved. One third $(34 \% ; n=258)$ went on to have at least one subsequent work-related injury in the following 24 months. Of those whose sentinel work-related injury was related to 'lifting/loading/pulling', $19 \%$ had at least one subsequent work-related injury event also related to this type of task. Predictors examined included pre-sentinel injury job strain, social support from colleagues and supervisors, physical work tasks, job security and job satisfaction.

Conclusion A substantial proportion of participants with a work-related sentinel injury had further work-related injuries in the following 24 months. The identification of factors that predict those at risk of subsequent work-related injuries could provide a useful focus for those involved in the rehabilitation of people with work-related injuries. 